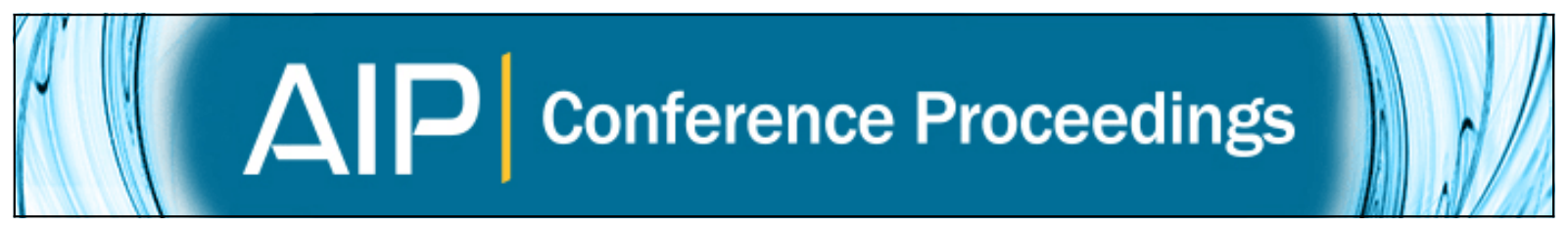

\title{
X-ray emission in the Outer Galaxy: the Star Forming Region NGC 1893
}

M. Caramazza, E. Flaccomio, L. Prisinzano, L. Rebull, S. Sciortino, J. R. Stauffer, S. Wolk, and G. Micela

Citation: AIP Conference Proceedings 1094, 580 (2009); doi: 10.1063/1.3099179

View online: http://dx.doi.org/10.1063/1.3099179

View Table of Contents: http://scitation.aip.org/content/aip/proceeding/aipcp/1094?ver=pdfcov

Published by the AIP Publishing

Articles you may be interested in

Extended X-ray emission in radio galaxies: 3C 305

AIP Conf. Proc. 1248, 473 (2010); 10.1063/1.3475306

X-ray emission from star forming regions

AIP Conf. Proc. 1248, 3 (2010); 10.1063/1.3475268

The rich low-mass population of the massive cluster NGC 6231: X-ray emission, evolutionary status, and IMF AIP Conf. Proc. 1094, 916 (2009); 10.1063/1.3099267

Modeling X-ray emission from stellar coronae

AIP Conf. Proc. 1094, 600 (2009); 10.1063/1.3099184

Hard X-ray emissions from star forming regions

AIP Conf. Proc. 393, 175 (1997); 10.1063/1.52782 


\title{
X-ray emission in the Outer Galaxy: the Star Forming Region NGC 1893
}

\author{
M. Caramazza ${ }^{* \dagger}$, E. Flaccomio ${ }^{\dagger}$, L. Prisinzano ${ }^{\dagger}$, L. Rebull**, S. Sciortino ${ }^{\dagger}$, \\ J.R. Stauffer**, S. Wolk ${ }^{\ddagger}$ and G. Micela ${ }^{\dagger}$ \\ *Dipartimento di Scienze Fisiche ed Astronomiche, Università degli studi di Palermo, Via Archirafi \\ 36, 90123 Palermo, Italy \\ ${ }^{\dagger}$ INAF Osservatorio Astronomico di Palermo, Piazza del Parlamento 1, 90134 Palermo, Italy \\ ${ }^{* *}$ Spitzer Science Center, Caltech 314-6, Pasadena, CA 91125 \\ ${ }^{\ddagger}$ Harvard-Smithsonian Center for Astrophysics, 60 Garden Street, Cambridge, MA 02138
}

\begin{abstract}
A key issue of the star formation process is its independence from the environmental conditions. In particular, it is not clear whether star formation in the outer Galaxy occurs in the same way as in the inner Galaxy. We present preliminary results of the analysis of a $\sim 440 \mathrm{Ks}$ ACISChandra observation of NGC1893, a young cluster ( 3-4Myrs), far away from the Galactic Center with the aim to study star formation in the outer region of the Galaxy and investigate the coronal properties of the cluster stars. We detect more than $1000 \mathrm{X}$-ray sources, most of which are likely cluster members. We present also a preliminary analysis of X-ray variability of the cluster stars.
\end{abstract}

Keywords: Stars: activity, coronae, flare, pre-main sequence, late-type - X-ray: stars

PACS: 97.10.Jb, 97.10.Bt, 97.21.a+,98.20.Di

\section{INTRODUCTION}

We know that the environmental conditions in the outer Galaxy should be not favorable to star formation: the average surface and volume densities of atomic and molecular hydrogen nebulae are much smaller than in the inner Galaxy [1], the interstellar radiation field is weaker [2], the prominent spiral arms are lacking and there are few supernovae that can trigger the star formation. Nevertheless, we do observe stars forming in the outer Galaxy and a key question is wether stars formation proceeds in the same way under these different conditions.

In order to investigate on this issue, we studied the properties of the young cluster NGC 1893, whose galactocentric distance is $>11 \mathrm{Kpc}$. We present the preliminary results of an ACIS/CHANDRA observation of the cluster, as part of the joint ChandraSpitzer large project The Initial Mass Function in the Outer Galaxy: the star forming region NGC1893 aimed at studying star formation processes at large galactocentric distances using multi-wavelength data, including infrared, X-ray, and optical observations.

\section{OBSERVATION}

The X-ray observation of NGC 1893 combines four nearly consecutive exposures of the cluster taken in 2006 November and a fifth exposure taken in 2007 January, with a total exposure time of $\sim 440 \mathrm{ks}$. A so long observation, is ideal for studies of short 


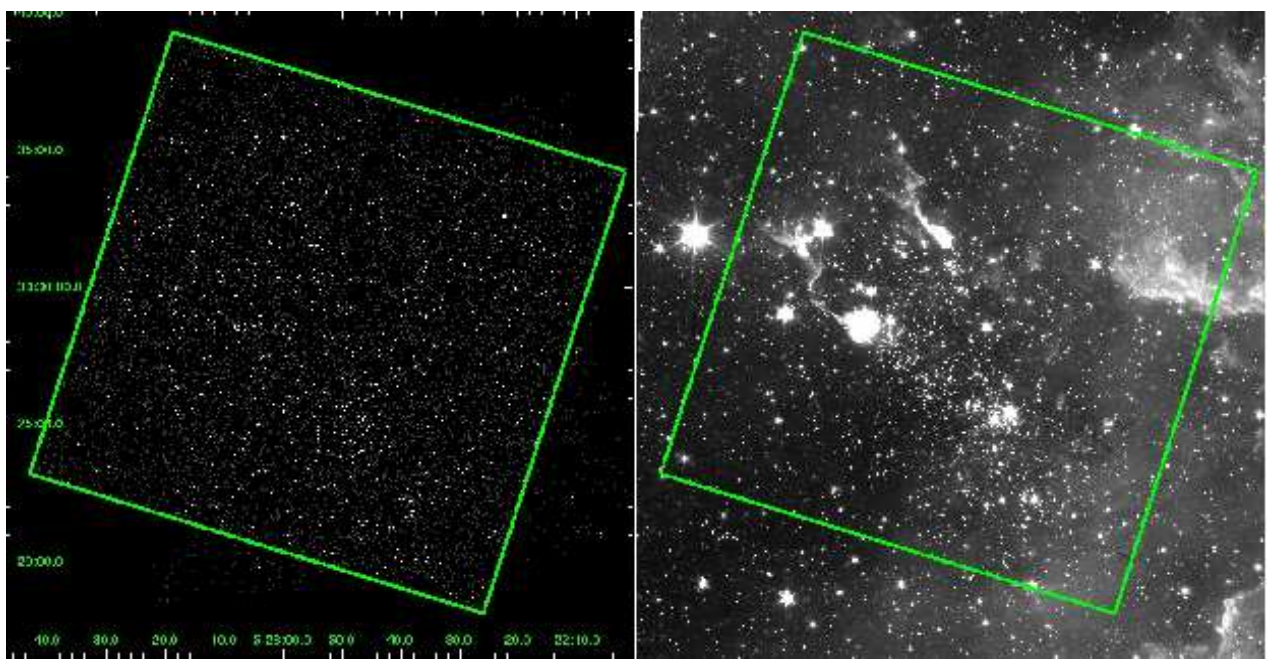

FIGURE 1. Chandra X-ray (left) and 4.5 micron Spitzer (right) images of NGC 1893. Green boxes mark the ACIS field of view

and medium period variability. The observations were obtained with the ACIS-I camera on-board Chandra [3, 4], covering $17 \mathrm{~min} \times 17 \mathrm{~min}$ in the sky (Fig. 1). The high angular resolution, with an on-axis PSF FWHM of $\sim 0.5 \mathrm{sec}$, makes it possible to resolve the central region of the cluster, despite the the crowdness due to the large distance of NGC 1893.

\section{X-RAY DETECTION AND CROSS IDENTIFICATION WITH IRAC SOURCES}

We detected sources applying the PWDetect code [5] to the merged event file of the five X-ray observations, filtering photons with energy between 0.5 and $8.0 \mathrm{KeV}$. After manual rejection of detections due to artifacts, we finally accepted 1021 distinct sources. Properties of X-ray sources were derived using the ACIS-Extract (AE) code [6].

We combined the Spitzer [7] and Chandra data, cross identifying the sources with three different matching radii $(0.7 \mathrm{sec}, 1.5 \mathrm{sec}, 2.5 \mathrm{sec})$, depending on the source off-axis angle. Considering the infrared sources detected in all the four IRAC energy bands, we find 227 matches that we consider as candidate members of the cluster. By means of the IRAC color-color diagram, we distinguish 116 stars, whose infrared emission is dominated by disk, 1 object with strong envelope emission and 110 sources in the color-color region corresponding to photospheres of normal stars. We consider these 110 sources to be Class III members of the cluster thanks to their high X-ray luminosity.

\section{X-RAY VARIABILITY}

We studied the variability of our sample, taking account also of different subsamples derived from the infrared classification [7]. 


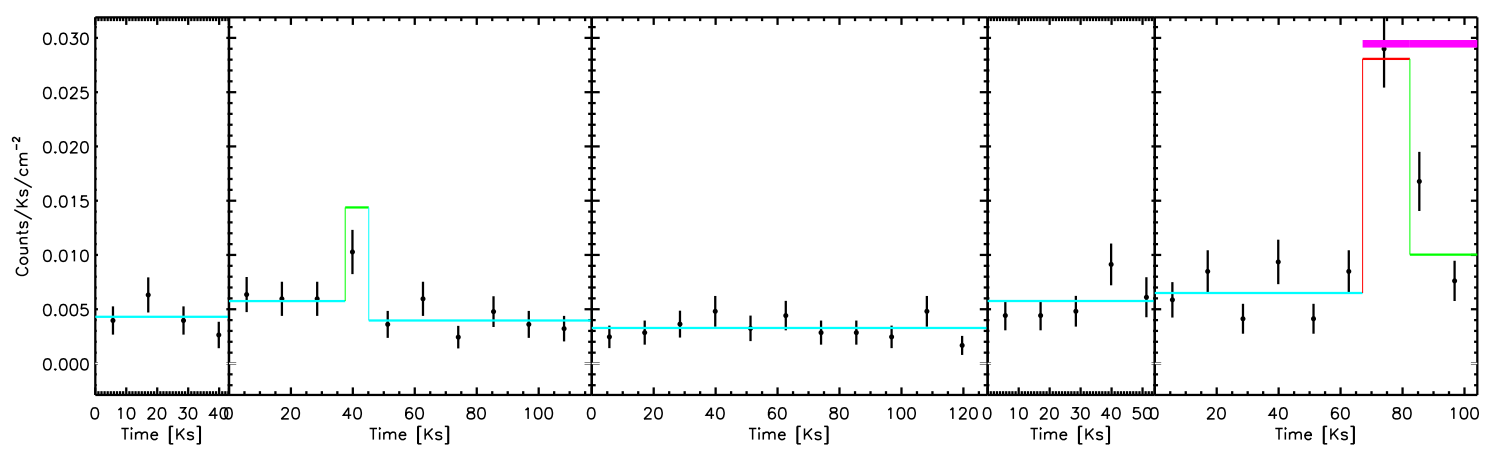

FIGURE 2. Example of light curve with a flare. The cyan blocks represent the "Characteristic level", the green blocks the elevated level, and the red block the very elevated level (see text). The flare is marked with a magenta line.

First, we analyzed the global variability of the light curves, applying the KolmogorovSmirnov test to the arriving times of photons, and obtained that $20 \%$ of our source are variable with a probability greater than $90 \% .23 \%$ of Class II stars are variable and $24 \%$ of the class III sources are variable.

We also analyzed the variability due to flares. In order to identify flares we use the method described in Caramazza et al. [8] based on two characteristics of flares: high count rate and its rapid variation. We first divide the light curve (Fig. 2) in blocks of constant count rate and classify them in characteristic blocks, compatible with the emission that the source keeps for most of the time, elevated blocks, slightly above the characteristic level and not usually associated to the macroscopic flares and the very elevated blocks, that combined with a high variation of count rate characterize flares.

In our sample of 1021 sources, 319 show flares. We found a frequency of flares of $\sim 0.3$ flares per source, that is the same of the subsample of Class II stars; in the Class III subsample we found a fraction of $\sim 0.2$ flares per source.

To investigate the mechanism of emission of X-ray sources, we studied the energy distribution of flares. The distribution is compatible with a power law (Fig. 3) with index $-2.6 \pm 0.2$ (grey line). For the subsample of Class II we found a slope of $-2.8 \pm 0.4$, while for Class III we found $-2.6 \pm 0.5$.

\section{PRELIMINARY CONCLUSIONS}

The sources of NGC1893 appear to be very active, a significant fraction of them show variability, and, in the entire sample we detected 319 flares during the five days of observation.

Studying the energy distribution of the observed flares, we noted that the distribution is compatible with a power law, with index $-2.6 \pm 0.2$. This slope is consistent with a model in witch the light curve is entirely due to overlapping flares and even the quiescent level is built from the superposition of microflares.

We compared the distributions of the entire sample with that of the subsamples of Class I and Class II stars, and we do not observe any significant difference among them. 


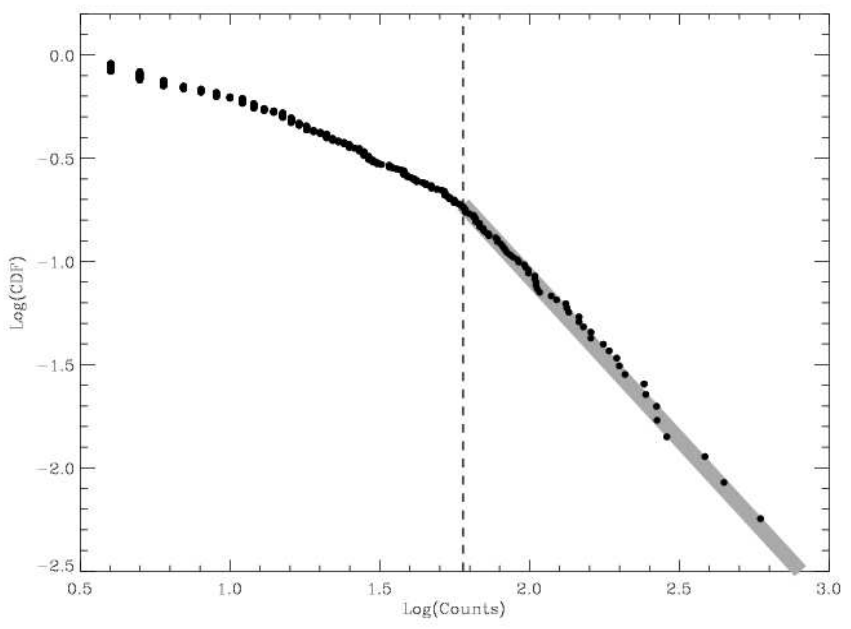

FIGURE 3. Cumulative probability function of flare intensities. Above the completeness limit (dashed line) the distribution is compatible with a power law; for low counts the distribution appears to saturate, probably because low counts flares are not detectable.

\section{ACKNOWLEDGMENTS}

We acknowledge financial contribution from contract ASI-INAF I/023/05/0 and from European Commission (contract N. MRTN-CT-2006-035890). This work is based on observations made with the Chandra Telescope.

\section{REFERENCES}

1. J. G. A. Wouterloot, J. Brand, W. B. Burton, and K. K. Kwee, $A \& A$ 230, 21-36 (1990).

2. J. S. Mathis, P. G. Mezger, and N. Panagia, $A \& A$ 128, 212-229 (1983).

3. M. C. Weisskopf, B. Brinkman, C. Canizares, G. Garmire, S. Murray, and L. P. Van Speybroeck, PASP 114, 1-24 (2002), arXiv:astro-ph/0110308

4. G. P. Garmire, M. W. Bautz, P. G. Ford, J. A. Nousek, and G. R. Ricker, Jr., "Advanced CCD imaging spectrometer (ACIS) instrument on the Chandra X-ray Observatory," in X-Ray and Gamma-Ray Telescopes and Instruments for Astronomy. Edited by Joachim E. Truemper, Harvey D. Tananbaum. Proceedings of the SPIE, Volume 4851, pp. 28-44 (2003)., edited by J. E. Truemper, and H. D. Tananbaum, 2003, vol. 4851 of Presented at the Society of Photo-Optical Instrumentation Engineers (SPIE) Conference, pp. 28-44.

5. F. Damiani, A. Maggio, G. Micela, and S. Sciortino, ApJ 483, 370-+ (1997).

6. P. Broos, L. Townsley, K. Getman, and F. Bauer, ACIS Extract, An ACIS Point Source Extraction Package, Pennsylvania State University (2002), http://WWW.astro.psu.edu/xray/docs/TARA/ae_users_guide.html

7. M. Caramazza, G. Micela, L. Prisinzano, L. Rebull, S. Sciortino, and J. R. Stauffer, A\&A 488, 211-218 (2008).

8. M. Caramazza, E. Flaccomio, G. Micela, F. Reale, S. J. Wolk, and E. D. Feigelson, A\&A 471, 645-654 (2007), arXiv:0706.1500 